\title{
THE SWEDISH RATING LIST
}

\author{
ICCA Communication
}

\section{$T$ Karlsson and G Grottling}

Contınuing our policy, this issue contains the latest version of the Swedish Ratıng List All games were played at 40 moves in two hours by members of the Swedish Chess Computer Association (SSDF) The ' + ' denotes the upward $95 \%$ confidence interval, the '-' denotes the downward $95 \%$ confidence interval on the ratıng, 'games' stands for the number of games on which the ratıng is based and 'against' stands for the average ratıng of opponents By elementary methods wider $(>95 \%)$ or narrower $(<95 \%)$ confidence intervals may be derived by referrıng to Gaussian statıstıcs

\begin{tabular}{|c|c|c|c|c|c|c|c|}
\hline \# & Name & ratıng & + & - & games & win \% & against \\
\hline 1 & Rebel 80 Pentium $90 \mathrm{MHz}$ & 2462 & 26 & 25 & 781 & 67 & 2337 \\
\hline 2 & Hiarcs 50 Pentium $90 \mathrm{MHz}$ & 2438 & 44 & 41 & 283 & 65 & 2331 \\
\hline 3 & M_Chess Pro 60 Pentium $90 \mathrm{MHz}$ & 2435 & 26 & 25 & 754 & 61 & 2356 \\
\hline 4 & Genius 50 Pentıum $90 \mathrm{MHz}$ & 2419 & 30 & 29 & 565 & 59 & 2353 \\
\hline 5 & Rebel 60 Pentium $90 \mathrm{MHz}$ & 2412 & 31 & 30 & 540 & 60 & 2340 \\
\hline 6 & Genıus 40 Pentıum $90 \mathrm{MHz}$ & 2411 & 25 & 25 & 809 & 64 & 2312 \\
\hline 6 & M_Chess Pro 50 Pentium $90 \mathrm{MHz}$ & 2411 & 26 & 25 & 759 & 62 & 2321 \\
\hline 8 & Genius 30 Pentrum $90 \mathrm{MHz}$ & 2410 & 27 & 26 & 706 & 62 & 2327 \\
\hline 9 & Rebel 70 Pent1um $90 \mathrm{MHz}$ & 2408 & 27 & 26 & 703 & 61 & 2327 \\
\hline 10 & Hiarcs 40 Pentium $90 \mathrm{MHz}$ & 2393 & 26 & 26 & 716 & 55 & 2357 \\
\hline 11 & Chessinaster 5000 Pentium $90 \mathrm{MHz}$ & 2386 & 49 & 45 & 240 & 67 & 2261 \\
\hline 12 & Nimzo 30 Pentium $90 \mathrm{MHz}$ & 2383 & 26 & 26 & 735 & 57 & 2332 \\
\hline 13 & Nimzo 35 Pentium $90 \mathrm{MHz}$ & 2382 & 48 & 48 & 215 & 50 & 2383 \\
\hline 14 & Hiarcs 30 Pentium $90 \mathrm{MHz}$ & 2379 & 30 & 30 & 545 & 56 & 2334 \\
\hline 14 & Genius $40486 / 5066 \mathrm{MHz}$ & 2379 & 28 & 28 & 628 & 57 & 2328 \\
\hline 16 & Junio1 3335 Pentium $90 \mathrm{MHz}$ & 2366 & 37 & 37 & 356 & 50 & 2365 \\
\hline 17 & $\mathrm{R} 30 \mathrm{v} 25$ & 2365 & 48 & 45 & 241 & 66 & 2249 \\
\hline 18 & M_Chess Pro 40 Pentium $90 \mathrm{MHz}$ & 2360 & 29 & 29 & 578 & 52 & 2344 \\
\hline 19 & Genıus $30486 / 5066 \mathrm{MHz}$ & 2358 & 24 & 23 & 910 & 62 & 2271 \\
\hline 20 & Fritz 30 Pentium $90 \mathrm{MHz}$ & 2354 & 26 & 26 & 735 & 53 & 2334 \\
\hline 21 & M_Chess Pro $50486 / 5066 \mathrm{MHz}$ & 2343 & 29 & 28 & 596 & 54 & 2317 \\
\hline 22 & Fritz 40 Pentıum $90 \mathrm{MHz}$ & 2336 & 41 & 40 & 304 & 59 & 2270 \\
\hline 23 & Rebel $70486 / 5066 \mathrm{MHz}$ & 2335 & 27 & 27 & 675 & 52 & 2324 \\
\hline 24 & Kallisto 198 Pentıum $90 \mathrm{MHz}$ & 2334 & 28 & 28 & 621 & 48 & 2349 \\
\hline 25 & WChess 106 Pentıum $90 \mathrm{MHz}$ & 2324 & 27 & 27 & 658 & 47 & 2345 \\
\hline 26 & M_Chess Pro $40486 / 5066 \mathrm{MHz}$ & 2314 & 24 & 24 & 846 & 53 & 2290 \\
\hline 27 & Chess Machine Schroder $313032 \mathrm{MHz}$ & 2302 & 32 & 30 & 546 & 68 & 2171 \\
\hline 27 & WChess 103 486/50 $66 \mathrm{MHz}$ & 2302 & 27 & 27 & 677 & 49 & 2308 \\
\hline 29 & Hiarcs $30486 / 5066 \mathrm{MHz}$ & 2300 & 24 & 24 & 865 & 50 & 2297 \\
\hline 29 & Rebel $60486 / 5066 \mathrm{MHz}$ & 2300 & 24 & 23 & 884 & 55 & 2265 \\
\hline 31 & Mephisto Genıus $68030 / 33 \mathrm{MHz}$ & 2299 & 47 & 46 & 228 & 57 & 2252 \\
\hline 32 & Chess Machine The King 20 aggr $\mathrm{R} 30$ off $30 \mathrm{MHz}$ & 2295 & 21 & 20 & 1182 & 65 & 2189 \\
\hline 33 & Chessmaster $4000486 / 5066 \mathrm{MHz}$ & 2292 & 34 & 33 & 462 & 66 & 2172 \\
\hline 34 & Rebel Decade Pentium $90 \mathrm{MHz}$ & 2281 & 29 & 30 & 563 & 45 & 2318 \\
\hline 35 & Fritz $30486 / 5066 \mathrm{MHz}$ & 2256 & 22 & 22 & 1040 & 50 & 2253 \\
\hline 36 & Comet 32 Pentrum $90 \mathrm{MHz}$ & 2224 & 32 & 33 & 488 & 33 & 2346 \\
\hline 37 & Berlın Pro $6802024 \mathrm{MHz}$ & 2223 & 25 & 24 & 816 & 58 & 2163 \\
\hline 38 & Kallisto $182183486 / 5066 \mathrm{MHz}$ & 2217 & 22 & 22 & 1021 & 43 & 2268 \\
\hline 39 & Kasparov Sparc $20 \mathrm{MHz}$ & 2213 & 25 & 25 & 758 & 51 & 2203 \\
\hline 40 & Saitek RISC 2500 ARM2 $14 \mathrm{MHz} 128 \mathrm{~K}$ & 2192 & 22 & 21 & 1062 & 56 & 2148 \\
\hline 41 & Mephisto Montreux ARM $14 \mathrm{MHz} 512 \mathrm{~K}$ & 2187 & 32 & 30 & 589 & 73 & 2015 \\
\hline 42 & Socrates $30486 / 33 \mathrm{MHz}$ & 2141 & 49 & 50 & 203 & 47 & 2162 \\
\hline 43 & Mephisto Berlın $6800012 \mathrm{MHz}$ & 2117 & 23 & 23 & 943 & 58 & 2063 \\
\hline 44 & Novag Sapphire H8 $10 \mathrm{MHz}$ & 2086 & 23 & 23 & 945 & 49 & 2096 \\
\hline 45 & Gandalf 21 Pentıum $90 \mathrm{MHz}$ & 2041 & 47 & 52 & 230 & 27 & 2214 \\
\hline 46 & Nimzo $221486 / 33 \mathrm{MHz}$ & 2034 & 46 & 47 & 229 & 42 & 2088 \\
\hline 47 & Zarkov $30486 / 2533 \mathrm{MHz}$ & 2029 & 46 & 48 & 232 & 39 & 2108 \\
\hline 48 & Kasparov Brute Force $\mathrm{H} 810 \mathrm{MHz}$ & 2018 & 23 & 23 & 906 & 44 & 2058 \\
\hline 49 & Complete Chess System $486 / 33 \mathrm{MHz}$ & 1984 & 47 & 47 & 221 & 47 & 2006 \\
\hline 50 & Kasparov President/GK $2100 \mathrm{H} 810 \mathrm{MHz}$ & 1976 & 29 & 30 & 558 & 47 & 1998 \\
\hline
\end{tabular}

As of January 1, 1996, the Swedish Rating List is no longer avallable to subscribers in printed form, though it will continue to be included in each issue of the Journal Those desirous to obtain updated copies of the Swedish Rating List are invited to extract them by FTP via the SSDF's Homepage of the Internet, http //www nsc liu se/ bosj/SSDF/ 